\title{
MAGE-A expression in oral and laryngeal leukoplakia predicts malignant transformation
}

\author{
Christoph A. Baran ${ }^{1} \cdot$ Abbas Agaimy $^{2} \cdot$ Falk Wehrhan $^{1} \cdot$ Manuel Weber $\mathbb{D}^{1} \cdot$ Verena Hille $^{1} \cdot$ Kathrin Brunner $^{5} \cdot$ \\ Claudia Wickenhauser $^{3}$. Udo Siebolts ${ }^{3} \cdot$ Emeka Nkenke $^{4} \cdot$ Marco Kesting $^{1} \cdot$ Jutta Ries $^{1}$
}

Received: 15 October 2018 / Revised: 17 February 2019 / Accepted: 27 February 2019 / Published online: 1 April 2019

(c) United States \& Canadian Academy of Pathology 2019

\begin{abstract}
Leukoplakia is a potential precursor of oral as well as laryngeal squamous cell carcinoma. Risk assessment of malignant transformation based on the grade of dysplasia of leukoplakia often does not lead to reliable results. However, oral squamous cell carcinoma, laryngeal squamous cell carcinoma, and leukoplakia express single or multiple members of the melanomaassociated antigens A (MAGE-A) family, while MAGE-A are absent in healthy mucosal tissue. The present study aimed at determining if there is an association between the expression of MAGE-A in leukoplakia and malignant transformation to oral or laryngeal squamous cell carcinoma. Paraffin-embedded tissues of 205 oral and laryngeal leukoplakia, 90 corresponding tumors, and 40 healthy oral mucosal samples were included in the study. The grade of dysplasia of the leukoplakia samples was determined histopathologically. The leukoplakia samples were divided into lesions that transformed to oral and laryngeal squamous cell carcinoma $(n=91)$ and lesions that did not $(n=114)$ during a 5 years follow-up. The expression of MAGE-A3/6 and MAGE-A4 was analyzed by real-time RT-PCR. The expression of MAGE-A $1-4,6$, and 12 was determined by immunohistochemistry. A total of 59.3\% of the transforming leukoplakia expressed at least one of the examined antigens as opposed to an expression rate of 3.5\% of all non-transforming leukoplakia. There was no MAGE-A expression in healthy oral mucosa. The risk of malignant transformation was statistically significantly associated with MAGE-A expression in immunohistochemistry $(p<0.001)$ and real-time RT-PCR (MAGE-A3/6, $p=0.001$; MAGE-A4, $p=0.002$ ) analyses. There was no significant association between MAGE-A expression and the grade of dysplasia ("low-grade", D0/D1; "high-grade", D2/D3) in immunohistochemistry $(p=0.412)$ and real-time RT-PCR (MAGE-A3/6, $p=0.667$; MAGE-A4, $p=0.756$ ). It seems that the analysis of the MAGE-A expression profile may support the identification of leukoplakia at risk for malignant transformation. Therefore, efforts should be made to establish this analysis as a routine procedure in addition to conventional histopathology.
\end{abstract}

Head and neck squamous cell carcinomas, including the oral squamous cell carcinoma and the laryngeal squamous cell carcinoma are the most common tumor entities among

Supplementary information The online version of this article (https:// doi.org/10.1038/s41379-019-0253-5) contains supplementary material, which is available to authorized users.

Christoph A. Baran

ChristophBaran@gmx.de

1 Department of Oral and Maxillofacial Surgery, Erlangen University Hospital, Friedrich-Alexander University ErlangenNürnberg (FAU), Erlangen, Germany

2 Institute of Pathology, Erlangen University Hospital, FriedrichAlexander University Erlangen-Nürnberg (FAU), Erlangen, Germany malignant disorders of the head and neck and the frequency of their occurrence in the population is high [1,2]. Their prognosis still remains poor due to their frequent detection in advanced stages of disease [3-6]. Both types of tumor can develop with varying frequency from potentially precancerous lesions like leukoplakia [7,8]. The range of malignant transformation from leukoplakia to squamous

3 Institute of Pathology, Halle (Saale) University Hospital, MartinLuther University Halle-Wittenberg (MLU), Halle (Saale), Germany

4 Department of Oral and Maxillofacial Surgery, Vienna General Hospital (AKH Wien), Medical University of Vienna,

Vienna, Austria

5 Institute of Pathology, Hospital Weiden, Weiden, Germany 
cell carcinoma amounts from $0.13 \%$ to $34 \%$ for oral leukoplakia and from $0 \%$ to $64.7 \%$ for laryngeal leukoplakia, respectively [9-12].

Today, prediction of the malignant potential is traditionally based on the histomorphologically determined severity of dysplasia. It is postulated that with the increase of severity of dysplastic changes the risk of malignant transformation into squamous cell carcinoma rises [8-10, 13]. However, oral leukoplakia with absence of dysplastic changes show malignant transformation in up to $16 \%$ and laryngeal leukoplakia with absence of dysplasia show malignant transformation rates of up to $3.7 \%$ of the cases $[8,14]$. Also spontaneous regression of severe dysplastic altered lesions has been observed [15-17]. Additionally, the exact determination of the grade of dysplasia depends on a subjective pathological assessment and is difficult to reproduce due to a large degree of inter- and intra-examiner variability [18, 19]. However, objective and reliable markers apart from the determination of the severity of dysplasia for the risk assessment of malignant transformation are missing [20,21]. Therefore, methods for an early detection of high-risk lesions and risk assessment of patients with leukoplakia are needed. In the past, several specific molecular markers have been described to be possible tools to predict the behavior of leukoplakia. However, out of these none found entry into routine clinical diagnostics yet because of their low sensitivity, low specificity and their limited reproducibility of observed results as described in previous clinical trials [22-25].

The melanoma-associated antigen A (MAGE-A) expression is exclusively restricted to testis, placenta tissues, and a number of solid malignant tumors, including oral squamous cell carcinoma and laryngeal squamous cell carcinoma. It is completely absent in healthy mucosal tissues [26-33]. It was already demonstrated that at least one of the 10 members of the MAGE-A family is expressed in $93 \%$ of oral squamous cell carcinoma tissues and in $46.2 \%$ of laryngeal squamous cell carcinoma tissues, whereas no expression could be observed in healthy oral mucosa $[29,30,34]$. Additionally, the expression of MAGE-A could be detected in oral precancerous lesions including oral leukoplakia. Furthermore, an association between the occurrence of MAGE-A and malignant transformation of oral leukoplakia was already assigned $[22,23,33]$. There are only a few studies investigating the MAGE-A expression profile in oral precursor lesions and malignant transformation to oral squamous cell carcinoma. Additionally, these studies only included a small number of patients. Investigations of the MAGE-A expression profile in laryngeal precursor lesions and malignant transformation to laryngeal squamous cell carcinoma do not exist.

Therefore, the aim of this study was to check whether the detection of MAGE-A is a useful tool to identify oral and laryngeal leukoplakia with high risk of an imminent malignant transformation.

\section{Material and methods}

\section{Material}

\section{Tissue samples and patient collective}

The study was approved by the Ethics Committee of the University of Erlangen-Nürnberg, Erlangen, Germany (approval number: 3962) and performed in accordance with the Declaration of Helsinki.

In total, 342 formalin-fixed paraffin-embedded tissues of a historical patient collective collected between 1994 and 2014 were provided from the Department of Pathology, University Hospital Erlangen and from the Department of Pathology, University Hospital Halle (Saale). The samples were located either in the oral cavity or in the larynx.

In the following, the term leukoplakia symbolizes a clinically visible white plaque on the mucosa that cannot be wiped away and cannot be attributed to other diseases or disorders that carry no increased risk for cancer [17]. Further histological specifications of clinically apparent leukoplakia are subsequently given as the histologically determined grade of epithelial dysplasia.

Specimens classified as healthy oral mucosa, oral leukoplakia, laryngeal leukoplakia (summarized as leukoplakia), proliferative verrucous leukoplakia, and oral and laryngeal squamous cell carcinoma (summarized as squamous cell carcinoma) were used in this study. There is evidence that erythroplakia and speckled leukoplakia harbor more often precancerous changes than conventional leukoplakia and even reveal the highest malignant potential among potentially precancerous lesions [17]. In this study none of the examined oral and laryngeal leukoplakia appeared clinically to be erythroplakic or speckled.

Samples were divided into 4 groups:

Group 1 consisted of 91 progressing leukoplakia that proceeded into squamous cell carcinoma within a time period of 5 years. The sample material was obtained by biopsy.

Group 2 consisted of 114 non-progressing leukoplakia that did not proceed into squamous cell carcinoma within a time period of 5 years. The sample material was obtained by biopsy.

Group 3 consisted of 90 corresponding squamous cell carcinoma tissues of the previously mentioned precursor lesions in group 1 . The sample material was obtained during tumor resection or if available by biopsy prior to tumor removal.

Group 4 consisted of 47 healthy oral mucosal tissues that were obtained from healthy volunteers during minor surgery and served as controls.

Out of these samples 48 progressive (group 1), 50 nonprogressive oral leukoplakia specimens (group 2), 25 
A

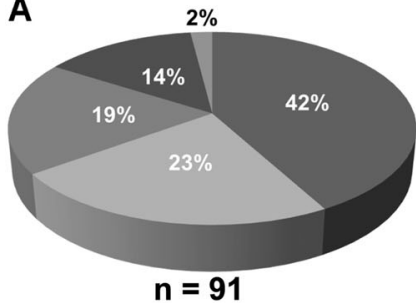

D

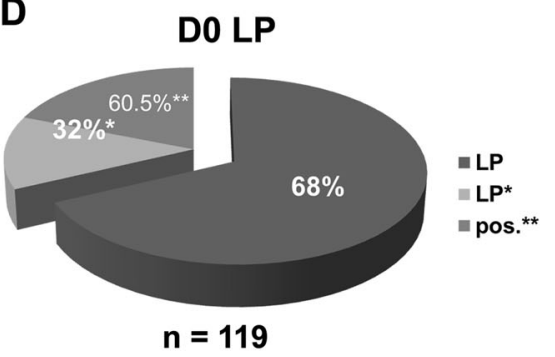

Fig. 1 Distribution of leukoplakia into the different grades of dysplasia including proliferative verrucous leukoplakia $(\mathbf{a}-\mathbf{c})$. Percentages of D0 and D1 leukoplakia within progressing (group 1) and non-progressing (group 2) leukoplakia and identification of transforming leukoplakia through MAGE-A expression (d, e). a Distribution of grades of dysplasia including proliferative verrucous leukoplakia within progressing leukoplakia (group 1). b Distribution of grades of dysplasia including proliferative verrucous leukoplakia within progressing (group 1) and non-progressing leukoplakia (group 2). c Distribution of grades of dysplasia including proliferative verrucous leukoplakia within nonprogressing leukoplakia (group 2). d Percentages of progressing (LP*) and non-progressing (LP) leukoplakia within investigated D0

healthy oral mucosal tissues (group 4), and 36 corresponding oral squamous cell carcinoma tissues (group 3) provided from the Department of Pathology, University Hospital Erlangen, already found entry into investigations of MAGE-A 1-4, 6, and 12 expression performed by immunohistochemistry. About 24 progressive (group 1), 50 non-progressing oral leukoplakia specimens (group 2), 30 healthy oral mucosal tissues (group 4), and 18 corresponding oral squamous cell carcinoma tissues (group 3) additionally already were investigated for the expression of MAGE-A1, 3, 4, 6, 10, and 12 performed by real-time RTPCR. Above-mentioned results from immunohistochemical as well from molecular biological investigations have already been published in 2012 [22, 23] and can be considered as preliminary work. In order to expand cases of progressive oral leukoplakia, corresponding oral squamous cell carcinoma tissues and non-progressive oral leukoplakia, additional cases from the Department of Pathology, University Hospital Erlangen, between 2011 and 2014 were included. To demonstrate the utility of MAGE-A expression in immunohistochemical and molecular biological analyses as a reliable predictive marker for risk assessment in leukoplakia, progressive and non-progressive oral and laryngeal leukoplakia and corresponding oral and laryngeal
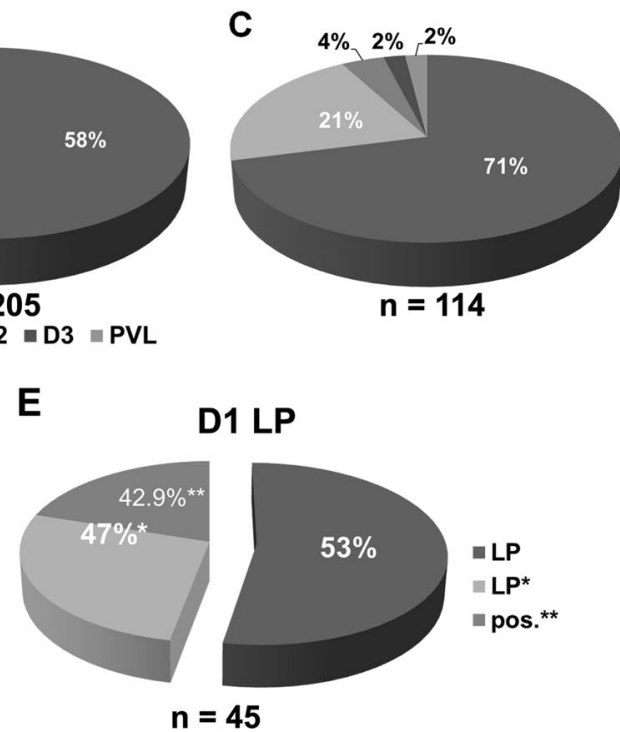

leukoplakia (group 1 and group 2) and detection of the upcoming malignant transformation through MAGE-A expression (pos.**). e Percentages of progressing (LP*) and non-progressing (LP) leukoplakia within investigated D1 leukoplakia (group 1 and group 2) and detection of the upcoming malignant transformation through MAGE-A expression (pos.**). PVL proliferative verrucous leukoplakia, LP percentage of non-progressing leukoplakia within all investigated D0 (D) and D1 (E) leukoplakia. LP* percentage of progressing leukoplakia within all investigated D0 (D) and D1 (E) leukoplakia, Pos.** percentage of the detection of the upcoming malignant transformation in D0 (D) and D1 (E) leukoplakia through MAGE-A expression

squamous cell carcinoma specimens between 1994 and 2014 were added from the Department of Pathology, University Hospital Halle (Saale), and investigated in a bicentric setting. The actual results substantiate the results already obtained in earlier investigations for MAGE-A expression as a molecular marker for risk assessment in oral leukoplakia $[22,23]$ and show the transferability and utility of the analysis of the MAGE-A expression profile in immunohistochemical and molecular biological analyses also in risk assessment for an upcoming malignant transformation in laryngeal leukoplakia.

\section{Clinical and histopathological data of healthy oral mucosa, leukoplakia, and tumor specimens}

All specimens were evaluated by three independent pathologists to ensure consistent results. Tissue samples in group 1 and 2 were histomorphologically classified as D0 for no, D1 for mild, D2 for moderate, and D3 for severe epithelial dysplasia according to the guidelines of the World Health Organization classification of tumors of the head and neck (2005) and were grouped as "low-grade" (D0/D1) precursor lesions standing for a potential "lowrisk" of malignant transformation and "high-grade" 
(D2/D3) precursor lesions standing for a potential "highrisk" of malignant transformation. For the diagnosis of proliferative verrucous leukoplakia, histological criteria like dense subepithelial lichenoid chronic inflammatory infiltrate, wavy hyperorthokeratosis, and a warty exophytic configuration were used [35]. Thus, the lesions included in this study in the category of proliferative verrucous leukoplakia were not compatible with simple hyperkeratotic or keratotic lesions. Due to the assessment of the grade of dysplasia already completed in 2014 and the statistic evaluation largely completed before the appearance of the current guidelines in 2017, the $3^{\text {rd }}$ Edition of the guidelines of the World Health Organization classification of tumors of the head and neck (2005) was used for histopathological grading. The distributions of grades of dysplasia are shown in Fig. 1a-c. Furthermore, the disease free survival which corresponds to the time interval between diagnosis of leukoplakia (group 1) and occurrence of malignancy (group 3) was ascertained. All invasive squamous cell carcinomas were characterized for grading (differentiation), TNM classification and clinical UICC-stage (I-IV) according to the $7^{\text {th }}$ Edition of the guidelines of the World Health Organization and the International Union Against Cancer $[36,37]$. Lymph node status (N-status) was grouped as N0 for absent lymph node metastases and $\mathrm{N}+$ for existent lymph node metastases. Additionally, tumor specimens were classified as well (G1), moderate (G2), and poor (G3) differentiated. The clinical UICC-stage (I-IV) was determined and grouped as "early" (I+II) and "late" (III+IV) stages. Since tumor size and lymph node involvement were not histopathologically determined in the majority of laryngeal squamous cell carcinomas, the indication of the TNM and the clinical UICC-stage was not possible for all examined cases. Healthy oral mucosal tissues were only used as controls if the absence of any epithelial dysplastic changes and/or local inflammation was histologically confirmed.

\section{Methods}

\section{Detection of MAGE-A expression by immunohistochemistry}

Immunohistochemistry was performed by the StreptAvidin-Biotin method using the pan-specific monoclonal antibody 57B (provided by Prof. Spagnoli, Basel, Switzerland) recognizing the antigens MAGE-A 1-4, 6, and 12. 2 - $\mu$ m-thick tissue cuttings were dewaxed using xylene for $45 \mathrm{~min}$ and rehydrated gradually. Subsequently, the slices were treated with a heat-based antigen retrieval method by using an EDTA-buffer ( $\mathrm{pH} 9.0 ; 10 \mathrm{mM}$ ) at $100^{\circ} \mathrm{C}$ for 10 min. Staining was done using a "Dako Cytomation
Autostainer Plus" (Dako Diagnostika GmbH, Hamburg, Germany) according to the manufacturer's specifications.

The staining procedure in brief was as follows. Before addition of the primary antibody, the tissue slides were incubated in an Avidin solution (Dako X9590) for $15 \mathrm{~min}$, rinsed with wash buffer (Dako S3006; pH 7.6), and treated with a Biotin solution in order to avoid the possibility of false reaction of endogenous biotin. Then a protein block with serum-free buffer (Dako X0909) was performed in order to elude non-specific binding reactions. The antibody was diluted (1:20) with antibody diluent (Dako S2022) and applied to each sample for $60 \mathrm{~min}$ at room temperature. The detection of the primary antibody was carried out by using a boitinylated anti-mouse/anti-rabbit-secondary reagent (Solution A; Dako REALTM detection system K5005), followed by the application of an alkaline-phosphate-streptavidin-biotin-complex system (Solution B; Dako REAL ${ }^{\mathrm{TM}}$ detection system K5005) and Fast Red as chromogen. Next, a counterstaining with haemalum (Dako REALTM S3301) was accomplished manually. Afterwards the slides were dried and covered with "Aquatex ${ }^{\circledR}$ (Merck Chemicals GmbH, Darmstadt, Germany).

Finally, all stained tissues were digitalized using the method of "whole slide imaging" in cooperation with the Institute of Pathology of the University of ErlangenNürnberg using a Panoramic 250 Flash III Scanner (3D Histech, Budapest, Hungary) in $\times 40$ magnification. Subsequently, all specimens were digitally analyzed (Case viewer, 3D Histech, Budapest, Hungary). Screenshots of single relevant areas of regions of interest were generated in $\times 10$ and $\times 20$ magnifications for assessment of immunohistochemistry and documentation. Nuclear and/or cytoplasmic staining was defined as positive result. Specimen of human testis was used as positive control in each staining run.

\section{RNA isolation and expression analysis of MAGE-A3/6 and MAGE-A4 by nested real-time RT-PCR}

Prior to isolation all paraffin blocks were trimmed after assessment and selection of suitable tissue areas to ensure consistent proportions of at least $75 \%$ of tissue areas of interest for investigation. Isolation from paraffin-embedded tissues was performed using the "RNeasy FFPE kit" (Qiagen, Hilden, Germany) according to the manufacturer's specifications. RNA quality and quantity were measured using the "NanoDrop 1000" (PeqLab Technologies, Erlangen, Germany) according to the manufacturers's instructions. For RT-PCR analysis a total of $100 \mathrm{ng}$ for MAGE-A expression analysis and a total of $50 \mathrm{ng}$ for GAPDH expression analysis as internal control for RNA integrity were used. Isolated RNA from formalin-fixed paraffinembedded human testis served as positive control. In the 
A

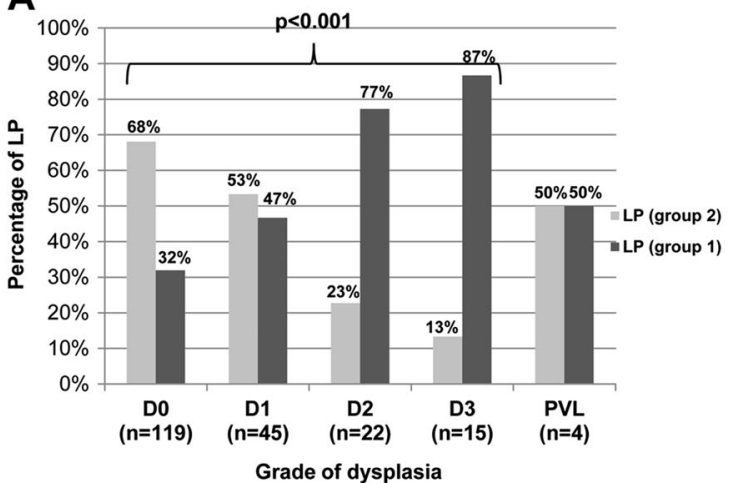

B

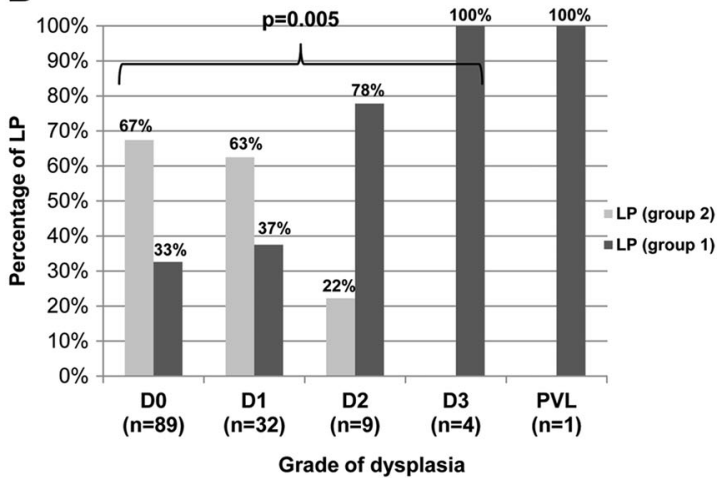

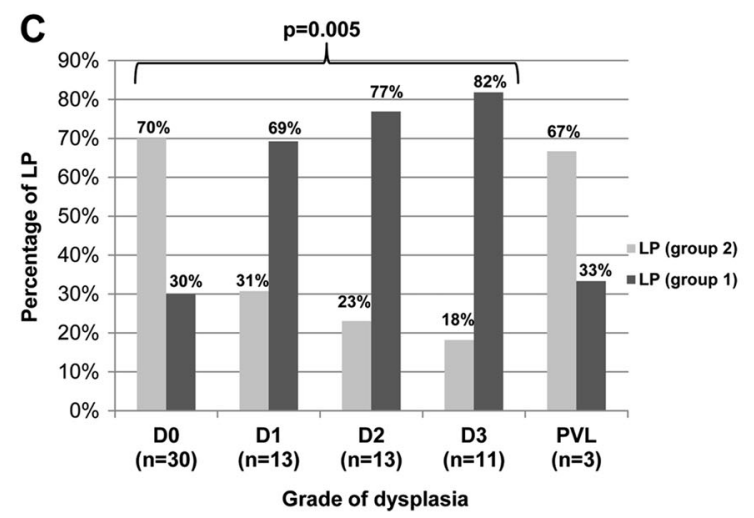

Fig. 2 Percentages of malignant transformation in relation to the grade of dysplasia including proliferative verrucous leukoplakia within progressive and non-progressive leukoplakia (group 1 and group 2). a Percentages of malignant transformation in relation to the grade of dysplasia including proliferative verrucous leukoplakia within investigated oral and laryngeal leukoplakia. b Percentages of malignant transformation in relation to the grade of dysplasia including

first step reverse transcription and the first amplification were carried out using the "One step RT-PCR kit" (Qiagen, Hilden, Germany) as indicated by the manufacturer. For this amplification step specific outer primers for the amplification of MAGE-A3/6, MAGE-A4, and GAPDH were used. The amplification was performed using the "PTC-200 Peltier Thermal Cycler" (Bio Rad MJ Research, Hercules, California, USA) and the thermal cycler "2720 Thermal Cycler" (Applied Biosystems; Weltham, Massacusetts, USA) for reverse transcription. Cycling conditions were as follows. In the first step, RT-PCR activation was carried out at $50{ }^{\circ} \mathrm{C}$ for $30 \mathrm{~min}$ and denaturation was done at $95^{\circ} \mathrm{C}$ for $15 \mathrm{~min}$, followed by 30 cycles of $94^{\circ} \mathrm{C}$ for $30 \mathrm{~s}$ (denaturation reaction), $60^{\circ} \mathrm{C}$ for $30 \mathrm{~s}$ (primer annealing reaction), and $72{ }^{\circ} \mathrm{C}$ for $60 \mathrm{~s}$ (elongation reaction). The same procedure was used for reverse transcription of GAPDH with the difference of using 20 cycles for RT-PCR instead of 30 .

All products from the first PCR reaction were diluted (1:10 for MAGE-A1-6; 1:100 for GAPDH) and a total volume of $1 \mu \mathrm{l}$ of these solutions for each specimen was used as template for highly sensitive second nested RT-PCR proliferative verrucous leukoplakia exclusively for leukoplakia located in the oral cavity. $\mathbf{c}$ Percentages of malignant transformation in relation to the grade of dysplasia including proliferative verrucous leukoplakia exclusively for leukoplakia located in the larynx. PVL proliferative verrucous leukoplakia. LP (group 2); non-progressing leukoplakia. LP (group 1); progressing leukoplakia

reaction using the "Power SYBR ${ }^{\circledR}$-Green PCR kit" (Applied Biosystems, Weiterstadt, Germany). The detection of specific mRNA was carried out using the "ABI Prism 7300 Sequence Detection System" (Applied Biosystems, Weiterstadt, Germany) with gene-specific inner primers for nested real-time RT-PCR amplification were used.

Cycling conditions for nested RT-PCR were as follows. Initial denaturation/enzyme activation was performed at $95^{\circ} \mathrm{C}$ for $10 \mathrm{~min}$, followed by 40 cycles of denaturation at $95^{\circ} \mathrm{C}$ for $15 \mathrm{~s}$ and annealing/elongation reaction at $60{ }^{\circ} \mathrm{C}$ for $60 \mathrm{~s}$. Each nested RT-PCR study was performed in duplicates. To evaluate the formation of the right amplification product, melting curve analysis was performed in each PCR reaction $[22,23]$. Cycle number 30 was defined as cut-off to indicate positive or negative MAGE-A3/6 and MAGE-A4 expression.

\section{Statistics}

For evaluation of the results, the statistical software package SPSS 21 (SPSS Inc., Chicago, Illinois, USA) was used. 
Table 1 Associations of MAGE-A, MAGE-A3/6, and MAGE-A 4 expression and the localization in the oral cavity and the larynx in progressing leukoplakia investigated by immunohistochemistry and real-time RT-PCR

\begin{tabular}{|c|c|c|c|c|c|c|c|c|c|c|c|c|c|c|}
\hline \multirow[b]{3}{*}{ Localization } & \multicolumn{8}{|c|}{ RT-PCR } & \multicolumn{4}{|c|}{ IHC } & \multirow[t]{3}{*}{ DFS } & \multirow[b]{3}{*}{$p$-value } \\
\hline & \multicolumn{4}{|c|}{ MAGE-A3/6 } & \multicolumn{4}{|c|}{ MAGE-A4 } & \multicolumn{4}{|c|}{ MAGE-A } & & \\
\hline & $n$ & + & $\%$ of cases & $p$-value & $n$ & + & $\%$ of cases & $p$-value & $n$ & + & $\%$ of cases & $p$-value & & \\
\hline Oral & 43 & 8 & 18.6 & & 33 & 3 & 9.1 & & 53 & 33 & 62.3 & & 79 weeks & \\
\hline Laryngeal & 21 & 1 & 4.8 & 0.135 & 18 & 3 & 16.7 & 0.422 & 38 & 20 & 52.6 & 0.358 & 118 weeks & 0.036 \\
\hline
\end{tabular}

$R T$-PCR real-time RT-PCR, IHC Immunohistochemistry, DFS disease free survival, $p$-values less than 0.05 are written in bold

Associations between MAGE-A expression determined by nested RT-PCR and immunohistochemistry in precancerous lesions (group 1 and group 2), the grade of dysplasia and cancer development were analyzed by the chi-squared test $\left(\chi^{2}\right.$-test) or the Mann-Whitney $U$ test. Associations in expression analysis of MAGE-A3/6, MAGE-A4 and MAGE-A in group 3 and histopathological parameters (grading, tumor size, lymph node status and clinical UICCstage) were analyzed by the chi-squared test $\left(\chi^{2}\right.$-test) or the Mann-Whitney $U$ test. Demographic, clinical, and histopathological results were generated by the chi-squared test $\left(\chi^{2}\right.$-test) or the Mann-Whitney $U$ test. $P$-values less than 0.05 were considered to indicate statistical significance.

\section{Results}

\section{Clinical features, demographic, and histomorphological data of the collective}

In this study, 342 formalin-fixed paraffin-embedded tissues were examined in total for the expression of MAGE-A3/6 and MAGE-A4 by nested real-time RT-PCR, and for the expression of MAGE-A (1-4, 6 and 12) using the panspecific antibody MAGE-57B by immunohistochemistry.

In total, 91 of all cases in group 1 matched criteria of progressing oral and laryngeal leukoplakia into oral and laryngeal squamous cell carcinoma in a period of 5 years. Forty nine $(54 \%)$ and forty two $(46 \%)$ of these cases were provided from the Department of Pathology of the University Hospital in Erlangen and from the Department of Pathology of the University Hospital in Halle (Saale), respectively. Fifty three $(58 \%)$ precancerous lesions were located in the oral cavity and thirty eight $(42 \%)$ in the larynx. There were no statistical significant differences considering the localization in the oral cavity, the larynx, and the affiliation to precancerous specimen material from Erlangen or Halle (Saale) $(p=0.14)$. Sixty seven $(74 \%)$ patients were male and twenty four $(26 \%)$ were female. The gender distribution matched between included cases from Erlangen and Halle (Saale) $(p=0.971)$. The mean age amounted to 60 years (min: 33 years; max.:75 years/ SD \pm 9 years) at the time of diagnosis of leukoplakia for patients from Erlangen and 63 years (min: 41 years; max.: 92 years/ $\mathrm{SD} \pm 10$ years) for patients from Halle (Saale) with no significant difference between both centers $(p=0.154)$. All grades of dysplasia (D0-D3) were present. There was a majority of D0 and D1 (65\%) within transforming leukoplakia (Fig. 1a). Two leukoplakia were classified as proliferative verrucous leukoplakia. Malignant transformation was significantly associated $(p<0.001)$ with increasing grade of dysplasia when all leukoplakia were taken into account (group 1 and group 2) (Fig. 2a). The same significant results were observed when oral leukoplakia $(p=$ 0.005; Fig. 2b) and laryngeal leukoplakia ( $p=0.005$; Fig. 2c) were taken individually into account. In total the mean disease free survival amounted to 95 weeks and was not significantly different between patients from Erlangen (mean: 104 weeks) and Halle (Saale) (mean: 85 weeks) $(p=0.363)$. Additionally, the disease free survival was independent of the histopathologically determined grade of dysplasia $(p=0.409)$. There was a weak statistical significant difference concerning the mean disease free survival for oral leukoplakia (79 weeks) and laryngeal leukoplakia (118 weeks) ( $p=0.036$; Table 1$)$.

In total 114 of all cases in group 2 matched criteria of non-progressing oral and laryngeal leukoplakia in a period of 5 years. About fifty nine (52\%) and fifty five (48\%) of these cases were provided from the Department of Pathology of the University Hospital in Erlangen and from the Department of Pathology of the University Hospital in Halle (Saale), respectively. Eighty two (72\%) precancerous lesions were located in the oral cavity and thirty two (28\%) in the larynx. There was a significant difference considering the localization and the affiliation of leukoplakia samples to one of both centers $(p<0.001)$. Seventy one $(62 \%)$ of the patients were male and forty three $(38 \%)$ were female. The samples matched in gender between Erlangen and Halle (Saale) $(p=0.147)$. The mean age amounted to 53 years (min.: 23 years; max.: 81 years/ $S D \pm 13$ years) at the time of diagnosis of leukoplakia. The average age of patients from Erlangen was significantly higher compared to that of patients from Halle (Saale) $(p=0.008)$. All grades of dysplasia (D0-D3) were present. Two specimens were 

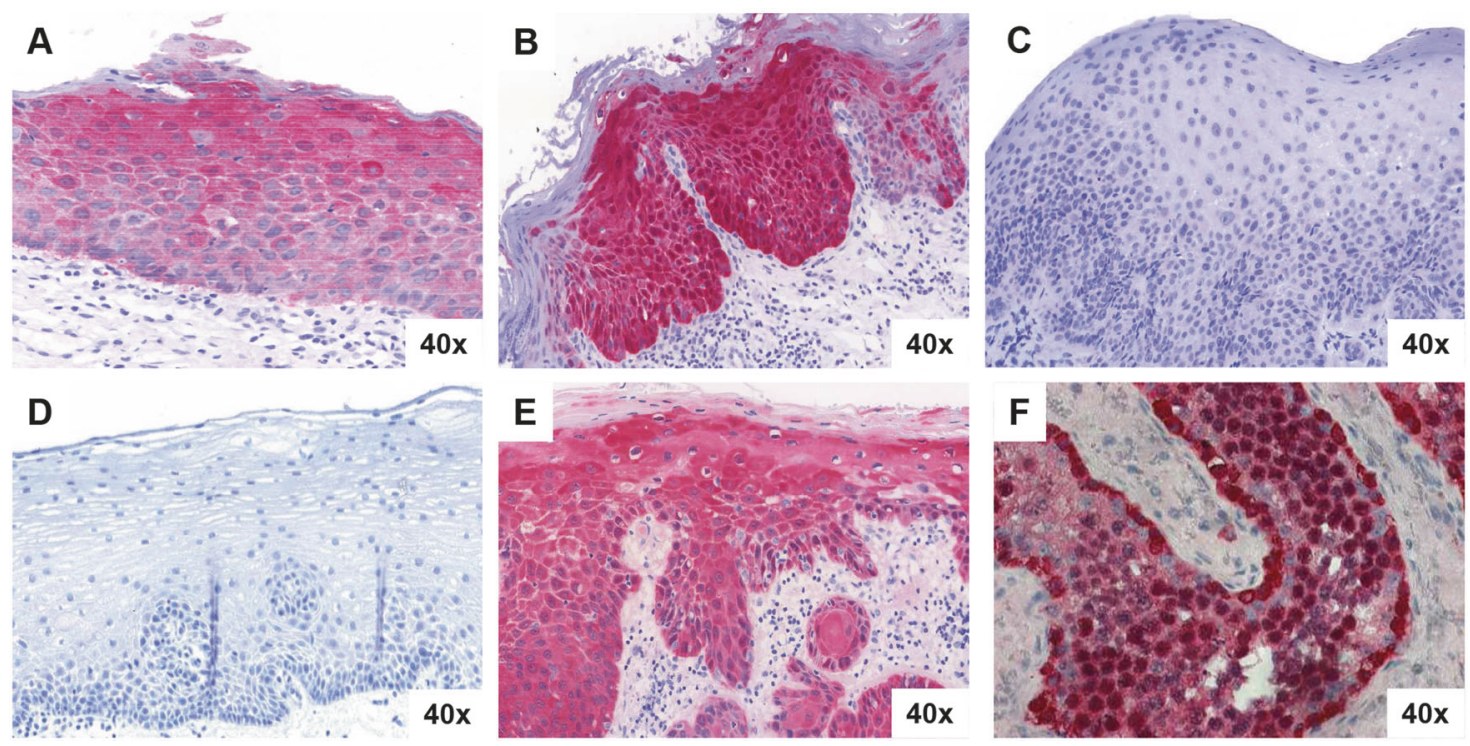

Fig. 3 Representative immunohistochemical staining results for MAGE-A in progressing leukoplakia, non-progressing leukoplakia, normal oral mucosa, corresponding tumor specimen and human testis (positive control). a Representative staining result of a progressing D0 leukoplakia (group 1) with MAGE-A expression. b Representative staining result of a progressing D2 leukoplakia (group 1) with MAGEA expression. c Representative staining result of a non-progressing D3 leukoplakia (group 2) with absent MAGE-A expression.

classified as proliferative verrucous leukoplakia. The mean observation period for non-progressing leukoplakia amounted to 593 weeks for patients from Erlangen and 747 weeks for patients from Halle (Saale). In total the mean period under review within non-progressing leukoplakia amounted to 667 weeks and was significantly higher for patients from Halle (Saale) $(p<0.001)$.

The comparison of group 1 and group 2 revealed significant differences in the mean age $(p<0.001)$, the localization in the oral cavity or the larynx $(p=0.04)$ and the distribution of grades of dysplasia $(p<0.001)$.

Group 3 (oral and laryngeal squamous cell carcinoma) consisted of 90 corresponding tumor specimens of the previously mentioned precursor lesions of group 1 .

Within group 1 and 3, eighty five (93\%) matching specimens, precursor lesions and corresponding tumors were available for investigation. In five $(6 \%)$ cases only the corresponding tumors of group 3 and in six (7\%) cases only the precursor lesions of group 1 were accessible for investigation. Tumor size (T-status) was available for fifty three $(59 \%)$ tumor samples. Six $(7 \%)$ tumor samples turned out to be carcinoma in situ (Cis). The corresponding lymph node status (N-status) was available for thirty eight (42\%) tumor samples. Additionally, the tumor grade was determined. All grades of differentiation (G1-G3) were present. Clinical UICC-stage was raised for forty one $(46 \%)$ patients. Healthy oral mucosal tissues from healthy volunteers in group 4 were used as controls d Representative staining result of normal oral mucosa (group 4) with absent MAGE-A expression. e Representative staining result of a MAGE-A positive corresponding tumor specimen (group 3). f Representative staining result of human testis (positive control) with MAGE-A expression. Nuclear and/or cytoplasmatic staining was considered to indicate positive MAGE-A expression. Illustrations were carried out in $\times 40$ magnification

based on the absence of any epithelial dysplastic changes and/or local inflammation. All healthy oral mucosal samples in group 4 originated from Erlangen and were located exclusively in the oral cavity.

\section{MAGE-A expression in leukoplakia, corresponding tumors, and healthy oral mucosa analyzed by immunohistochemistry}

In total, 330 specimens found entry into expression analyses performed by immunohistochemistry. Representative examples for positive and negative staining results of progressing and non-progressing leukoplakia, corresponding tumors, healthy oral mucosa, and human testis (positive control) are displayed in Fig. 3.

Within progressing leukoplakia in total fifty three (58.2\%) specimens showed MAGE-A expression, whereas thirty eight (41.8\%) showed no MAGE-A expression. Positive and negative cases were equally distributed among patients from Erlangen and Halle (Saale) $(p=0.14)$. Additionally, no significant differences concerning MAGEA expression and localization in the oral cavity or in the larynx were found ( $p=0.358$; Table 1$)$. Grouped grades of dysplasia as "low-risk" (D0/D1) and "high-risk" (D2/D3) dysplasia within progressing leukoplakia showed no significant difference concerning MAGE-A expression $(p=$ 0.412; Table 2). The estimation of an upcoming malignant transformation was possible in seventy two $(60.5 \%)$ cases 
Table 2 Associations of grouped grades of dysplasia and MAGEA, MAGE-A3/6, and MAGEA4 expression in progressing leukoplakia investigated by immunohistochemistry and realtime RT-PCR
Table 3 Associations of MAGEA, MAGE-A3/6 and MAGE-A 4 expression and histopathological characteristics in corresponding tumor specimens investigated by immunohistochemistry and realtime RT-PCR

\begin{tabular}{|c|c|c|c|c|c|c|c|c|c|c|c|c|}
\hline \multirow[b]{3}{*}{$\begin{array}{l}\text { Grade of dysplasia } \\
\text { (grouped) }\end{array}$} & \multicolumn{8}{|c|}{ RT-PCR } & \multirow{2}{*}{\multicolumn{4}{|c|}{$\frac{\mathrm{IHC}}{\text { MAGE-A }}$}} \\
\hline & \multicolumn{4}{|c|}{ MAGE-A3/6 } & \multicolumn{4}{|c|}{ MAGE-A4 } & & & & \\
\hline & $n$ & + & $\begin{array}{l}\% \text { of } \\
\text { cases }\end{array}$ & $p$-value & $n$ & + & $\begin{array}{l}\% \text { of } \\
\text { cases }\end{array}$ & $p$-value & $n$ & + & $\begin{array}{l}\% \text { of } \\
\text { cases }\end{array}$ & $p$-value \\
\hline D0/D1 & 45 & 6 & 13.3 & & 36 & 4 & 11.1 & & 59 & 32 & 54.2 & \\
\hline D2/D3 & 17 & 3 & 17.6 & & 14 & 2 & 14.3 & & 30 & 19 & 63.3 & \\
\hline D0/D1 vs. D2/D3 & & & & 0.667 & & & & 0.756 & & & & 0.412 \\
\hline
\end{tabular}

RT-PCR real-time RT-PCR, IHC immunohistochemistry

\begin{tabular}{|c|c|c|c|c|c|c|c|c|c|c|c|c|}
\hline & \multicolumn{8}{|c|}{ RT-PCR } & \multicolumn{4}{|c|}{$\mathrm{IHC}$} \\
\hline & \multicolumn{4}{|c|}{ MAGE-A3/6 } & \multicolumn{4}{|c|}{ MAGE-A4 } & \multicolumn{4}{|c|}{ MAGE-A } \\
\hline & $\bar{n}$ & + & $\%$ of cases & $p$-value & $n$ & + & $\%$ of cases & $p$-value & $n$ & + & $\%$ of cases & $p$-value \\
\hline \multicolumn{13}{|l|}{ Tumor size } \\
\hline 1 & 29 & 10 & 34.5 & & 23 & 3 & 13.0 & & 37 & 27 & 73.0 & \\
\hline 2 & 15 & 5 & 33.3 & & 7 & 4 & 57.1 & & 13 & 10 & 76.9 & \\
\hline 3 & 2 & 1 & 50 & & 2 & 1 & 50 & & 36 & 3 & 8.3 & \\
\hline 4 & 6 & 1 & 16.7 & & 3 & 1 & 33.3 & & 6 & 4 & 66.7 & \\
\hline Cis & 3 & 1 & 33.3 & 0.856 & 4 & 1 & 25.0 & 0.18 & 5 & 4 & 80.0 & 0.845 \\
\hline \multicolumn{13}{|c|}{ Tumor size (grouped) } \\
\hline $\mathrm{T} 1 / \mathrm{T} 2$ & 41 & 15 & 36.6 & & 30 & 7 & 23.3 & & 50 & 37 & 74.0 & \\
\hline $\mathrm{T} 3 / \mathrm{T} 4$ & 8 & 2 & 25.0 & 0.529 & 5 & 2 & 40.0 & 0.430 & 9 & 7 & 77.8 & 0.881 \\
\hline \multicolumn{13}{|c|}{ Lymph node status } \\
\hline No & 23 & 6 & 26.1 & & 15 & 4 & 26.7 & & 29 & 21 & 72.4 & \\
\hline $\mathrm{N}+$ & 7 & 3 & 42.9 & 0.343 & 5 & 2 & 40.0 & 0.483 & 7 & 6 & 85.7 & 0.426 \\
\hline \multicolumn{13}{|c|}{ Grade of differentiation } \\
\hline 1 & 16 & 5 & 31.3 & & 10 & 0 & 0 & & 18 & 12 & 66.7 & \\
\hline 2 & 35 & 16 & 45.7 & & 25 & 8 & 32.0 & & 43 & 33 & 76.7 & \\
\hline 3 & 12 & 4 & 33.3 & 0.546 & 9 & 3 & 33.3 & 0.115 & 17 & 12 & 70.6 & 0.868 \\
\hline \multicolumn{13}{|c|}{ Clinical UICC-stage } \\
\hline I/II (early) & 23 & 10 & 43.5 & & 15 & 4 & 26.7 & & 28 & 22 & 78.6 & \\
\hline III/IV (late) & 11 & 2 & 18.2 & 0.149 & 6 & 3 & 50.0 & 0.306 & 12 & 9 & 75.0 & 0.804 \\
\hline
\end{tabular}

IHC immunohistochemistry, RT-PCR real-time RT-PCR, Cis carcinoma in situ, NO without lymph node infestation, $N+$ with lymph node infestation among D0 leukoplakia (Fig. 1d) and in fifty one (42.9\%) cases among D1 leukoplakia (Fig. 1e) by occurrence of MAGE-A expression. Both examined proliferative verrucous leukoplakia which progressed into carcinoma showed MAGE-A expression. Disease free survival was independent of MAGE-A expression $(p=0.730)$.

Within non-progressing leukoplakia, in total, four specimens revealed MAGE-A expression. Both examined proliferative verrucous leukoplakia that did not progress into malignancy showed no MAGE-A expression.

In corresponding tumors no significant association between the T-status and MAGE-A expression was apparent ( $p=0.845$; Table 3$)$, even after grouping into "small" (T1/T2) and "large" (T3/T4) malignancies ( $p=0.811$; Table 3). Furthermore, there was no significant association concerning existent $(\mathrm{N}+)$ and absent $(\mathrm{N} 0)$ lymph node metastases and MAGE-A expression ( $p=0.426$; Table 3$)$. According to the grading, there was no significant association between the differentiation status and MAGE-A expression ( $p=0.868$; Table 3$)$. Additionally, no significant association between the grouped clinical UICC-stage for "early" (UICC-stages I and II) and "late" (UICC-stages III and IV) stages and MAGE-A expression $(p=0.804$; Table 3$)$ was found.

Within specimens of healthy oral mucosa, none of the 37 tissues showed MAGE-A expression.

Statistical analysis revealed that MAGE-A expression in progressing leukoplakia was significantly different compared to non-progressing leukoplakia $(p<0.001$; Table 4$)$. The same significant results were observed for comparison 
Table 4 Associations of MAGEA, MAGE-A3/6, and MAGEA4 expression in progressing leukoplakia (group 1), nonprogressing leukoplakia (group 2), corresponding tumor specimens (group 3 ) and normal oral mucosa (group 4) investigated by immunohistochemistry and realtime RT-PCR

\begin{tabular}{|c|c|c|c|c|c|c|c|c|c|c|c|c|}
\hline \multirow[b]{3}{*}{ Group } & \multicolumn{8}{|c|}{ RT-PCR } & \multirow{2}{*}{\multicolumn{4}{|c|}{$\frac{\mathrm{IHC}}{\mathrm{MAGE}-\mathrm{A}}$}} \\
\hline & \multicolumn{4}{|c|}{ MAGE-A3/6 } & \multicolumn{4}{|c|}{ MAGE-A4 } & & & & \\
\hline & $n$ & + & $\%$ of cases & $p$-value & $n$ & + & $\%$ of cases & $p$-value & $n$ & + & $\%$ of cases & $p$-value \\
\hline 1 & 64 & 9 & 14.1 & & 51 & 6 & 11.8 & & 91 & 53 & 58.2 & \\
\hline 2 & 90 & 0 & 0 & & 79 & 0 & 0 & & 114 & 4 & 3.5 & \\
\hline 1 vs. 2 & & & & 0.001 & & & & 0.002 & & & & 0.001 \\
\hline 1 & 64 & 9 & 14.1 & & 51 & 6 & 11.8 & & 91 & 53 & 58.2 & \\
\hline 4 & 40 & 0 & 0 & & 29 & 0 & 0 & & 37 & 0 & 0 & \\
\hline 1 vs. 4 & & & & 0.013 & & & & 0.055 & & & & 0.001 \\
\hline 2 & 90 & 0 & 0 & & 79 & 0 & 0 & & 114 & 4 & 3.5 & \\
\hline 4 & 40 & 0 & 0 & & 29 & 0 & 0 & & 37 & 0 & 0 & \\
\hline 2 vs. 4 & & & & n.d. & & & & n.d. & & & & 0.248 \\
\hline 1 & 64 & 9 & 14.1 & & 51 & 6 & 11.8 & & 91 & 53 & 58.2 & \\
\hline 3 & 69 & 26 & 37.7 & & 51 & 12 & 23.5 & & 88 & 63 & 71.6 & \\
\hline 1 vs. 3 & & & & 0.002 & & & & 0.119 & & & & 0.062 \\
\hline
\end{tabular}

$R T$-PCR real-time-RT-PCR, IHC immunohistochemistry, n.d. not determinable, $p$-values less than 0.05 are written in bold of MAGE-A expression between progressing leukoplakia and healthy oral mucosa $(p<0.001$; Table 4$)$. However, no significant difference concerning MAGE-A expression between non-progressing leukoplakia and healthy oral mucosa could be ascertained ( $p=0.248$; Table 4$)$. Moreover, MAGE-A expression was not significantly different between progressing leukoplakia and corresponding tumors $(p=0.062 ;$ Table 4$)$. Hence, expression of MAGE-A (specificity: 0.965) in tissues is highly specific for the detection of malignancy and progressing leukoplakia. Based on the calculated prevalence of $44.3 \%$ for progressing leukoplakia within all investigated leukoplakic lesions (group 1 and group 2), the resulting test accuracy amounted to a positive predictive value of $93.0 \%$ and a negative predictive value of $74.3 \%$. Comparing group 1 and 2, the sensitivity for the detection of progressing leukoplakia amounted to 58.2\% (positive: 53/91; negative: 38/91). Hence, occurring MAGE-A expression in progressing leukoplakia proved to be a highly reliable predictor for malignant transformation $(p<0.001)$.

\section{Expression of MAGE-A3/6 and MAGE-A4 in leukoplakia, corresponding tumors, and healthy oral mucosa analyzed by real-time RT-PCR}

In total, 263 specimens were available for expression analysis of MAGE-A3/6 and 210 specimens for expression analysis of MAGE-A4 by nested real-time RT-PCR. Representative results for expression analyses in progressing leukoplakia non-progressing leukoplakia, corresponding carcinomas, healthy oral mucosa, human testis (positive control), and GAPDH (internal control) are displayed, respectively, in Fig. 4.
Within progressing leukoplakia in nine (14.1\%) cases the expression of MAGE-A3/6 was detectable, whereas in fifty five $(85.9 \%)$ MAGE-A3/6 expression was missing. Concerning MAGE-A4 six (11.8\%) cases showed MAGE-A4 expression and forty five $(88.2 \%)$ cases showed no MAGEA4 expression. Progressing leukoplakia which showed MAGE-A4 expression were equally distributed among Erlangen and Halle (Saale) $(p=0.409)$, whereas MAGEA3/6 expression was significantly different between Erlangen and Halle (Saale) $(p=0.01)$. No significant differences for MAGE-A3/6 ( $p=0.135$; Table 1$)$ and MAGE-A4 ( $p=$ 0.422 ; Table 1) expression concerning the localization in the oral cavity or in the larynx were found.

Grouped grades of dysplasia as "low-risk" (D0/D1) and "high-risk" (D2/D3) dysplasia within progressing leukoplakia showed no significant difference concerning MAGEA3/6 $(p=0.667$; Table 2$)$ or MAGE-A4 $(p=0.756$; Table 2) expression. Disease free survival within group 1 was independent of whether MAGE-A3/6 $(p=0.078)$ or MAGE-A4 ( $p=0.281)$ was expressed.

Within non-progressing leukoplakia no lesion showed expression of MAGE-A3/6 or MAGE-A4, respectively. The resulting specificity for the detection of leukoplakia with absent risk of malignant transformation amounted to $100 \%$ for MAGE-A3/6 as well as for MAGE-A4.

Statistical investigations of the association between MAGE-A3/6 and MAGE-A4 expression and risk of malignant transformation in group 1 revealed high statistical significance for MAGE-A3/6 $(p<0.001$; Table 4$)$ and MAGE-A4 ( $p=0.002$; Table 4).

In group 3, there were no significant associations between the T-status and MAGE-A3/6 expression $(p=$ 0.856; Table 3) or MAGE-A4 $(p=0.18$; Table 3$)$, 
A

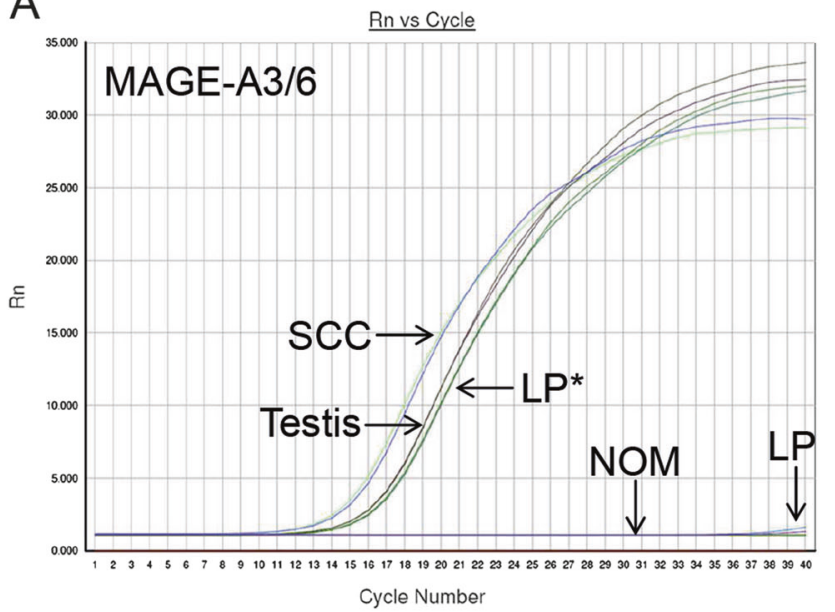

B

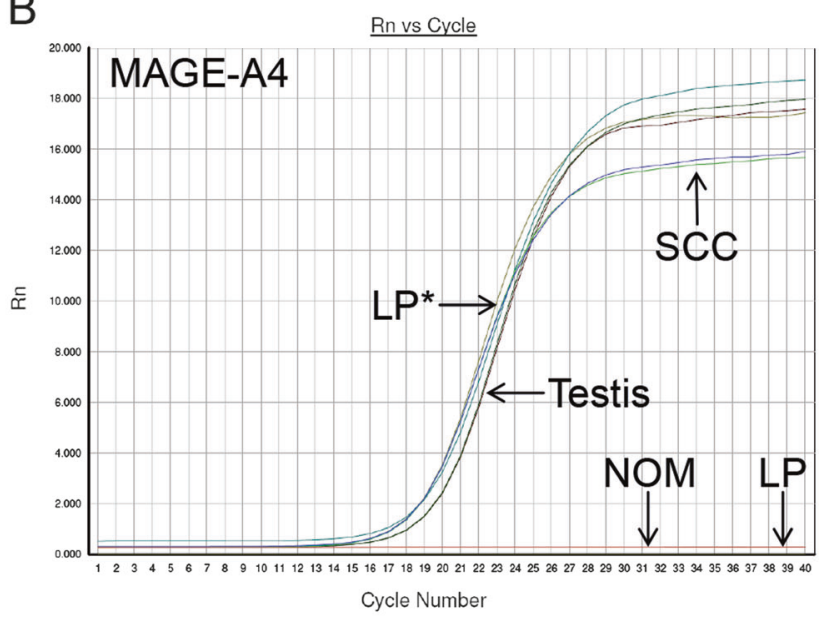

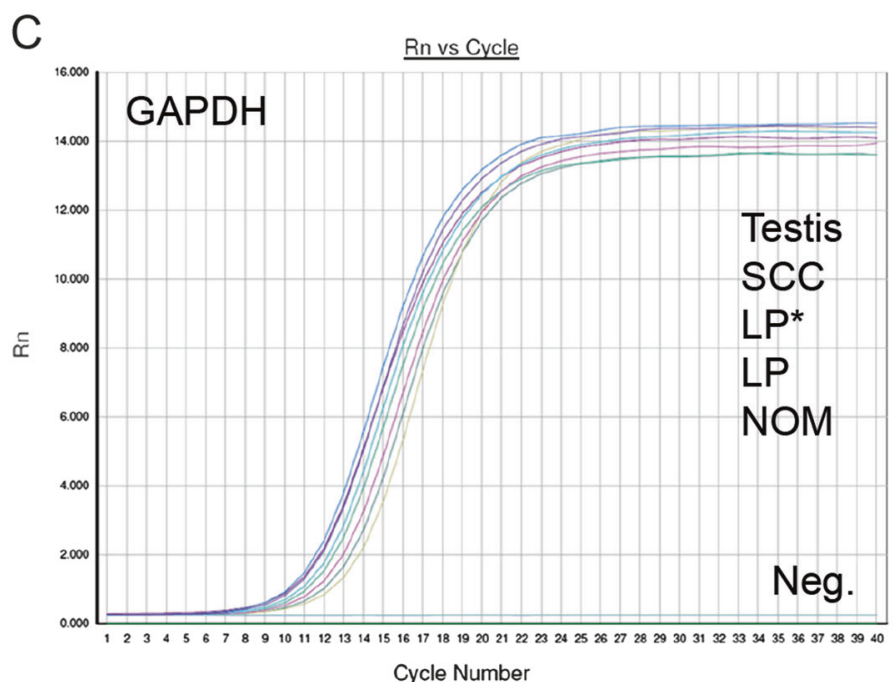

Fig. 4 Expression analysis of progressing leukoplakia, nonprogressing leukoplakia, corresponding squamous cell carcinoma, normal oral mucosal tissue, and human testis (positive control) carried out by RT-PCR. a Representative results of expression analyses for positive MAGE-A3/6 expression. b Representative results of expression analyses for positive MAGE-A4 expression. c The quality of

respectively, even after grouping into "small" (T1/T2) and "large" (T3/T4) malignancies (MAGE-A3/6: $p=0.529$; MAGE-A4: $p=0.430$; Table 3). No significant associations between absent (NO) and existent $(\mathrm{N}+)$ lymph node metastases and MAGE-A3/6 $(p=0.343$; Table 3$)$ or MAGE-A4 expression ( $p=0.483$; Table 3$)$ could be detected. The same applies for the differentiation status (grading) and MAGE-A3/6 ( $p=0.546$; Table 3 ) or MAGEA4 ( $p=0.115$; Table 3$)$ expression. Furthermore, we could not detect any significant associations between MAGE-A3/ 6 expression $(p=0.149$; Table 3$)$, MAGE-A4 expression $(p=0.306$; Table 3$)$, and grouped clinical UICC-stages.

Out of the investigated healthy oral mucosal tissues and non-progressing leukoplakia none expressed MAGE-A3/6 or MAGE-A4.
RNA was assessed by amplifying cDNA using GAPDH as internal control. LP* progressing leukoplakia (group 1), LP non-progressing leukoplakia (group 2), SCC squamous cell carcinoma (group 3), NOM normal oral mucosa (group 4), Testis human testis (positive control), Neg. negative control reaction without cDNA template

Statistical analysis revealed a significant difference between progressing leukoplakia and non-progressing leukoplakia for MAGE-A3/6 ( $p=0.001$; Table 4$)$ and MAGEA4 expression ( $p=0.002$; Table 4$)$. There was a significant difference between progressing leukoplakia and healthy oral mucosa $(p=0.013$; Table 4$)$ for MAGE-A3/6 but not for MAGE-A4 expression $(p=0.055$; Table 4$)$. A statistical significant difference concerning MAGE-A3/6 expression in progressing leukoplakia and corresponding tumors could be ascertained ( $p=0.002$; Table 4$)$. However, no significant difference between group 1 and group 3 could be demonstrated for MAGE-A4 expression $(p=0.119$; Table 4).

The sensitivity for the detection of progressing leukoplakia amounted to $14.1 \%$ (positive: $9 / 64$; negative: $55 / 64$ ) for MAGE-A3/6 and 11.8\% (positive: 6/51; negative: 45/ 
51) for MAGE-A4 expression. Values for specificity were $100 \%$ for MAGE-3/6 and $100 \%$ for MAGE-A4. The test accuracy revealed a positive predictive value of $100 \%$ for the detection of progressing leukoplakia and a negative predictive value of $62.1 \%$ for the actual discovery of nonprogressing leukoplakia within a time period of 5 years for MAGE-A3/6 and a positive predictive value of $100 \%$ and a negative predictive value of $63.7 \%$ for MAGE-A4.

\section{Combinations of MAGE-A expression by immunohistochemistry and MAGE-A3/6 and MAGE- A4 by real-time RT-PCR analyses as a "multi-marker system"}

When combining results for expression analyses for MAGE-A by immunohistochemistry and MAGE-A3/6 and MAGE-A4 by real-time RT-PCR as a "multi-marker system" the sensitivity amounted to $16.7 \%$ with a specificity of $100 \%$ for positive expression of all markers. The positive predictive value was up to $100 \%$ with a simultaneous negative predictive value of $83.5 \%$ for the combination of all markers. The combination of MAGE-A and MAGE-A3/ 6 expression revealed a sensitivity of $26.5 \%$ and a specificity of $100 \%$ with a resulting positive predictive value of $100 \%$ and a negative predictive value of $77.7 \%$. The sensitivity was $25.0 \%$ for the combination of MAGE-A and MAGE-A4 with a specificity of $100 \%$. The positive predictive value amounted to $100 \%$ with a negative predictive value of $83.9 \%$ for this composition. Finally a sensitivity of $7 \%$ with a specificity of $100 \%$ was calculated for concurrent expression of MAGE-A3/6 and MAGE-A4. The resulting positive value amounted to $100 \%$ with a negative predictive value of $65.8 \%$ for co-expression of MAGE-A3/6 and MAGE-A4 detected by real-time RT-PCR analyses.

\section{Discussion}

Up to date, risk assessment and management of premalignant oral and laryngeal lesions depend on clinical appearance and histopathological grading of epithelial dysplasia performed by single or serial incisional biopsy $[38,39]$. The utilization of different grading systems concerning architectural and cytological changes often does not lead to reliable results in the determination of the grade of dysplasia and the prediction of the malignant potential. Additionally, grading of epithelial dysplasia is essentially subjective and depends on the experience and the individual interpretation of the presence and the degree of histological changes. Both circumstances lead to a significant amount of intra- and inter-examiner variability $[18,19]$. Hence, reliable and objective prognostic parameters including molecular markers are needed as tools for risk assessment in oral and laryngeal leukoplakia [20, 21].
Although, many molecular biomarkers such as MAGE-A or the epithelial growth factor receptor are currently discussed to be useful tools for the diagnosis of oral and laryngeal squamous cell carcinoma, none of them found entry into clinical routine so far $[25,29-32,40-43]$. The expression of MAGE-A in healthy human tissues is exclusively restricted to testis and placenta tissues. However, its detection in various cancer entities including oral and laryngeal squamous cell carcinoma and even in precursor lesions of oral squamous cell carcinoma was already demonstrated [22, 23, 33]. Nevertheless, the above-mentioned studies which examined precursor lesions only included a small number of patients and additionally investigated only leukoplakia localized in the oral cavity to illustrate the reliability for its use as a diagnostic tool in clinical routine. In addition, these studies were restricted to a single cancer center. Therefore, the present study aimed to back up the usefulness of MAGE-A expression in oral leukoplakia by comparing precursor lesions which showed a malignant transformation within a time period of 5 years with lesions that did not show the development into carcinoma by increasing the number of oral leukoplakia cases. Moreover, leukoplakia localized in the larynx were investigated to check the applicability of MAGE-A expression as a predictive marker for malignant transformation also in laryngeal leukoplakia. To the best of our knowledge, the recent study is the first one to investigate a 5 years follow-up in oral as well as in laryngeal leukoplakia on such a large bi-centric collective. Additionally, to date investigations of MAGE-A expression in laryngeal leukoplakia in regard to the association with malignant transformation into laryngeal squamous cell carcinoma do not exist. The present study is the first one to analyze the expression of MAGE-A by immunohistochemistry and real-time RT-PCR in oral and laryngeal leukoplakia in parallel. Hence, in order to confirm the importance of expression analysis of MAGE-A to determine the risk of malignant transformation, we increased the number of cases of a previously in Erlangen performed and already published pre-random study with additional specimens of oral leukoplakia, corresponding oral squamous cell carcinomas, laryngeal leukoplakia, and corresponding laryngeal squamous cell carcinomas from two independent centers. By expanding the examinations on laryngeal leukoplakia, we expected new insights into tumor development on the basis of precursor lesions in the laryngeal area, too.

In this study, the statistical association between the risk of malignant transformation and increasing grade of dysplasia in oral as well as in laryngeal leukoplakia could be confirmed [8-10, 13]. Nevertheless, the proportion of low dysplastic D0 and D1 leukoplakia in the group of progressing lesions was high. Leukoplakia histopathologically assessed as D0 (41.8\%) and D1 (23.1\%) represent within progressing leukoplakia the majority in our study. 
Furthermore, within all D0 and D1 leukoplakia the proportion of D0 leukoplakia revealed transformation rates of $31.9 \%$ while transformation rates of $46.7 \%$ were even higher for D1 leukoplakia. This illustrates the major problem of a substantial and not negligible number of precancerous lesions that are judged as "low-risk" lesions by histomorphological assessment of dysplasia, but develop into carcinoma. It is now of primary importance to identify these precancerous lesions in order not to misjudge their malignant potential which could lead to a medical undersupply of the patient.

It could be shown that MAGE-A expression in progressing leukoplakia was not significantly associated to grouped grades of dysplasia histopathologically categorized as "low-risk" lesions (D0/D1), or D2 and D3 categorized as "high-risk" lesions. Hence, it could be claimed that the detection of leukoplakia, especially of D0 and D1 lesions at risk of malignant transformation could be detected in $60.5 \%$ of all progressing D0 leukoplakia and in $42.9 \%$ of all progressing D1 leukoplakia more reliable by MAGE-A expression than just by determination of the histopathologically determined grade of dysplasia. Additionally, two progressing and two non-progressing proliferative verrucous leukoplakia were investigated. As they represent a different and to this day not understood entity of unknown origin, different to conventional leukoplakia [35, 44], these four specimens were also analyzed. Since the determination of the grade of dysplasia in these lesions is not carried out by histological assessment, the association of MAGE-A expression performed by immunohistochemistry and RTPCR and grade of dysplasia for proliferative verrucous leukoplakia was not possible. Despite proliferative verrucous leukoplakia specimens are clearly underrepresented, interestingly both progressing proliferative verrucous leukoplakia expressed MAGE-A in immunohistochemistry, whereas both non-progressive proliferative verrucous leukoplakia showed no MAGE-A expression in immunohistochemistry. There were no histomorphological differences between both progressing and both non-progressing proliferative verrucous leukoplakia concerning abovementioned criteria of histological diagnosis. Therefore, it could be suggested that expression analyses of MAGE-A could serve as a prognostic tool in proliferative verrucous leukoplakia, too. However, it has to be mentioned that due to the small number of two progressing and two nonprogressing proliferative verrucous leukoplakia no cases of verrucous carcinoma were investigated. For that reason, no meaningful statement can be made regarding MAGE-A expression and malignant transformation to verrucous carcinoma of these lesions. This question needs to be evaluated in future studies comprising larger series of proliferative verrucous leukoplakia lesions and verrucous carcinoma but would go beyond the scope of the current study.
The disease free survival was not significantly associated with positivity for MAGE-A, as already described [22, 23]. This could be demonstrated for laryngeal leukoplakia, too. Hence, it could be assumed that there is no difference between oral and laryngeal leukoplakia concerning MAGEA expression and disease free survival. Furthermore, the disease free survival was independent of the grade of dysplasia. Moreover, within leukoplakia which did not show malignant transformation, only four specimens showed positivity exclusively for MAGE-A by immunohistochemistry. This result of non-transforming leukoplakia does not match findings in previous studies which have shown no expression of MAGE-A in non-progressing oral leukoplakia [23]. The reason for positive cases in the recent investigation is likely to be caused by the unclear data situation or the specific development of malignancy or tumor resection in another cancer center. However, the exact reason for MAGE-A positivity in these few cases remains unknown.

In the present study, we could not reproduce the already described percentage rates of $85.4 \%$ in progressing oral leukoplakia and $86.1 \%$ in corresponding tumors for MAGE-A expression determined by immunohistochemistry [23]. Our results amount to a sensitivity of $58.2 \%$ for the risk of an upcoming malignant transformation in leukoplakia and a percentage of $65.3 \%$ positive corresponding tumor specimens with a high specificity of $96.5 \%$. This difference to previous investigations is likely caused by the larger case number and a different distribution of grades of dysplasia, but not by the inclusion of leukoplakia located in the laryngeal area or by inclusion of cases from another cancer center as our results show. However, due to the limited sample material and the restricted sample size, expression analyses by real-time RT-PCR were selectively performed for the genes MAGE-A3/6 and MAGE-A4. These two genes of the MAGE-A family seem to be the most suitable genes for the diagnosis of oral squamous cell carcinoma and the prediction of malignant transformation of oral leukoplakia due to their highest expression rates in oral squamous cell carcinoma as determined in our previous research [22].

For expression analyses of MAGE-A3/6 and MAGE-A4 by real-time RT-PCR results for sensitivity were clearly lower than in immunohistochemistry. The reason for the lower sensitivity in RT-PCR could be that the pan-specific monoclonal antibody 57B recognizes the antigens MAGEA 1-4, 6, and 12 in immunohistochemistry, whereas in RTPCR analyses only the genes MAGE-A3/6 and MAGE-A4 were evaluated. Nevertheless, a high specificity of $100 \%$ could be reached for MAGE-A3/6 and MAGE-A4. On one hand, due the low expression rates of MAGE-A3/6 and MAGE-A4 in progressing leukoplakia, it seems that expression analyses of MAGE-A by immunohistochemistry are more reliable for the detection of leukoplakia at risk of an imminent malignant transformation. On the other hand, 
due to their high specificity, expression analyses of MAGEA3/6 and MAGE-A4 by RT-PCR could support as confirmatory test for the affirmation of an absent progression in cases of absent MAGE-A expression ascertained by immunohistochemistry in leukoplakia. The currently low sensitivity for the detection of leukoplakia at risk of malignant transformation determined by MAGE-A3/6 and MAGE-A4 expression in RT-PCR could be solved by adding and analyzing further genes of the MAGE-A family, as already described $[22,31]$.

Our results may have impact in patient-specific treatment. To date therapy strategies in management of leukoplakia focus on "watchfull waiting", non-surgical application of local therapeutics and photodynamic therapy, serial incision biopsies, or excision biopsy and laserevaporation depending on the determined grade of dysplasia and clinically apparent extension of the leukoplakia $[7,39,45,46]$. In the present study in more than half of transforming D0 and D1 leukoplakia the development into carcinoma could be detected by MAGE-A expression. Therefore, the detection of MAGE-A already in the early stages of D0 and D1 leukoplakia could indicate a more aggressive surgical treatment and closer inspection intervals [47-49]. Thus, undersupply due to a wait-and-see attitude and the inevitable misjudgment produced by local not representative incision biopsies that underdiagnose dysplastic lesions and even mask oral squamous cell carcinoma could be avoided [47]. In addition, no progress into carcinoma in more than $85 \%$ of non-transforming leukoplakia could be confirmed in D2 and D3 leukoplakia through absent MAGE-A expression. This observation could indicate a cautious attitude regarding surgical treatment and avoid overtreatment of leukoplakia. Expression analyses of melanoma-associated antigens could thus support to predict malignant transformation in histopathological assessment and help in therapy planning in oral and laryngeal leukoplakia. In connection with the treatment options another aspect should be mentioned.

It is known that antigen-specific target therapies and the polyvaccination against MAGE-A are already applied in various cancer entities and are discussed to be useful in oral squamous cell carcinoma therapy $[50,51]$. Due to their restricted expression in leukoplakia they appear to be ideal targets for specific immunotherapies in precancerous lesions, too. Hence, the expression analysis in precancerous lesions could possibly lead to new effective methods that counteract malignant transformation. This could ultimately lead to a reduction in the incidence and consequently to a decrease of the mortality of oral and laryngeal squamous cell carcinoma.

The suggestion of the authors is that further efforts in identifying conventional and proliferative verrucous oral and laryngeal leukoplakia with high risk of a malignant transformation need to be a central focus in prospective investigations with larger patient collectives in multi-center studies analyzing the whole panel of all MAGE-A family members in RT-qPCR in order to increase the sensitivity of the diagnostic method.

Acknowledgements All authors have read the manuscript and approved its submission. The present work was performed in (partial) fulfillment of the requirements for obtaining the degree "Dr. med. dent." at the Friedrich-Alexander-University Erlangen-Nürnberg (FAU). The authors thank the research technicians Mrs. E. Diebel and Mrs. S. Schönherr for the valuable support in assistance and performance in immunohistochemistry and Mrs. A. Krautheim-Zenk for the valuable support in assistance and performance in RNA isolation and RT-qPCR analyses.

\section{Compliance with ethical standards}

Conflict of interest The authors declare that they have no conflict of interest.

Publisher's note: Springer Nature remains neutral with regard to jurisdictional claims in published maps and institutional affiliations.

\section{References}

1. Ferlay J, Soerjomataram I, Dikshit R, et al. Cancer incidence and mortality worldwide: sources, methods and major patterns in GLOBOCAN 2012. Int J Cancer. 2015;136:E359-386.

2. Siegel RL, Miller KD, Jemal A. Cancer Statistics, 2018. CA Cancer J Clin. 2018;68:7-30.

3. Sundermann BV, Uhlmann L, Hoffmann J, Freier K, Thiele OC. The localization and risk factors of squamous cell carcinoma in the oral cavity: A retrospective study of 1501 cases.J Cranimaxillofac Surg. 2018;46:177-82.

4. Ong TK, Murphy C, Smith AB, et al. Survival after surgery for oral cancer: a 30-year experience. Br J Oral Maxillofac Surg. 2017;55:911-6.

5. Tribius S, Donner J, Pazdyka H, et al. Survival and overall treatment time after postoperative radio(chemo)therapy in patients with head and neck cancer. Head Neck. 2016;38:1058-65.

6. Jansen L, Buttmann-Schweiger N, Listl S, et al. Differences in incidence and survival of oral cavity and pharyngeal cancers between Germany and the United States depend on the HPVassociation of the cancer site. Oral Oncol. 2018;76:8-15.

7. Kuribayashi Y, Tsushima F, Morita KI, et al. Long-term outcome of non-surgical treatment in patients with oral leukoplakia. Oral Oncol. 2015;51:1020-5.

8. Isenberg JS, Crozier DL, Dailey SH. Institutional and comprehensive review of laryngeal leukoplakia. Ann Otol Rhinol Laryngol. 2008;117:74-79.

9. Warnakulasuriya S, Ariyawardana A. Malignant transformation of oral leukoplakia: a systematic review of observational studies. J Oral Pathol Med. 2016;45:155-66.

10. van Hulst AM, Kroon W, van der Linden ES, et al. Grade of dysplasia and malignant transformation in adults with premalignant laryngeal lesions. Head Neck. 2016;38:2284-90.

11. Scheifele C, Reichart PA. Oral leukoplakia in manifest squamous epithelial carcinoma. A clinical prospective study of 101 patients. Mund Kiefer Gesichtschir. 1998;2:326-30.

12. Reibel J. Prognosis of oral pre-malignant lesions: significance of clinical, histopathological, and molecular biological characteristics. Crit Rev Oral Biol Med. 2003;14:47-62. 
13. Weller MD, Nankivell PC, McConkey C, et al. The risk and interval to malignancy of patients with laryngeal dysplasia; a systematic review of case series and meta-analysis. Clin Otolaryngol. 2010;35:364-72.

14. Silverman S Jr., Gorsky M, Lozada F. Oral leukoplakia and malignant transformation. A follow-up study of 257 patients. Cancer. 1984;53:563-8.

15. Johnson NW, Jayasekara P, Amarasinghe AA. Squamous cell carcinoma and precursor lesions of the oral cavity: epidemiology and aetiology. Periodontol 2000. 2011;57:19-37.

16. Speight PM, Khurram SA, Kujan O. Oral potentially malignant disorders: risk of progression to malignancy. Oral Surg Oral Med Oral Pathol Oral Radiol. 2018;125:612-27.

17. Warnakulasuriya S, Johnson NW, van der Waal I. Nomenclature and classification of potentially malignant disorders of the oral mucosa. J Oral Pathol Med. 2007;36:575-80.

18. Krishnan L, Karpagaselvi K, Kumarswamy J, et al. A. Inter- and intra-observer variability in three grading systems for oral epithelial dysplasia. J Oralmaxillofac Pathol. 2016;20:261-8.

19. Speight PM, Abram TJ, Floriano PN, et al. Interobserver agreement in dysplasia grading: toward an enhanced gold standard for clinical pathology trials. Oral Surg Oral Med Oral Pathol Oral Radiol. 2015;120:474-82.

20. Pitiyage G, Tilakaratne WM, Tavassoli M, et al. Molecular markers in oral epithelial dysplasia: review. J Oral Pathol Med. 2009;38:737-52.

21. Rivera C, Oliveira AK, Costa RAP, et al. Prognostic biomarkers in oral squamous cell carcinoma: a systematic review. Oral Oncol. 2017;72:38-47.

22. Ries J, Agaimy A, Vairaktaris E, et al. Evaluation of MAGE-A expression and grade of dysplasia for predicting malignant progression of oral leukoplakia. Int J Oncol. 2012;41:1085-93.

23. Ries J, Agaimy A, Vairaktaris E, et al. Detection of MAGE-A expression predicts malignant transformation of oral leukoplakia. Cancer Invest. 2012;30:495-502.

24. Nankivell P, Weller M, McConkey C, et al. Biomarkers in laryngeal dysplasia: a systematic review. Head Neck. 2011;33: 1170-6.

25. Pereira CM, Gomes CC, De Fatima Correia Silva J, et al. Evaluation of MAGE A1 in oral squamous cell carcinoma. Oncol Rep. 2012;27:1843-8.

26. Ries J, Schultze-Mosgau S, Neukam F, et al. Investigation of the expression of melanoma antigen-encoding genes (MAGE-A1 to -A6) in oral squamous cell carcinomas to determine potential targets for gene-based cancer immunotherapy. Int $\mathbf{J}$ Oncol. 2005;26:817-24.

27. Mollaoglu N, Vairaktaris E, Nkenke E, et al. Expression of MAGE-A12 in oral squamous cell carcinoma. Dis Markers. 2008;24:27-32.

28. Muller-Richter UD, Dowejko A, Peters S, et al. MAGE-A antigens in patients with primary oral squamous cell carcinoma. Clin Oral Investig. 2010;14:291-6.

29. Han L, Jiang B, Wu H, et al. Expression and prognostic value of MAGE-A9 in laryngeal squamous cell carcinoma. Int Clin Exp Pathol. 2014;7:6734-42.

30. Liu S, Sang M, Xu Y, et al. Expression of MAGE-A1, -A9, -A11 in laryngeal squamous cell carcinoma and their prognostic significance: a retrospective clinical study. Acta Otolaryngol. 2016;136:506-13.

31. Ries J, Vairaktaris E, Mollaoglu N, et al. Expression of melanoma-associated antigens in oral squamous cell carcinoma. $\mathrm{J}$ Oral Pathol Med. 2008;37:88-93.
32. Figueiredo DL, Mamede RC, Spagnoli GC, et al. High expression of cancer testis antigens MAGE-A, MAGE-C1/CT7, MAGE-C2/ CT10, NY-ESO-1, and gage in advanced squamous cell carcinoma of the larynx. Head Neck. 2011;33:702-7.

33. Krauss E, Rauthe S, Gattenlohner S, et al. MAGE-A antigens in lesions of the oral mucosa. Clin Oral Investig. 2011;15:315-20.

34. Ries J, Mollaoglu N, Toyoshima T, et al. A novel multiple-marker method for the early diagnosis of oral squamous cell carcinoma. Dis Markers. 2009;27:75-84.

35. Gillenwater AM, Vigneswaran N, Fatani H, et al. Proliferative verrucous leukoplakia (PVL): a review of an elusive pathologic entity! Adv Anat Pathol. 2013;20:416-23.

36. Patel SG, Shah JP. TNM staging of cancers of the head and neck: striving for uniformity among diversity. CA Cancer J Clin. 2005;55:242-58.

37. Paleri V, Mehanna H, Wight RG. TNM classification of malignant tumours7th edition: what's new for head and neck? Clin Otolaryngol. 2010;35:270-2.

38. Warnakulasuriya $\mathrm{S}$, Reibel $\mathrm{J}$, Bouquot $\mathrm{J}$, et al. Oral epithelial dysplasia classification systems: predictive value, utility, weaknesses and scope for improvement. J Oral Pathol Med. 2008;37:127-33.

39. Karatayli-Ozgursoy S, Pacheco-Lopez P, Hillel AT, et al. Laryngeal dysplasia, demographics, and treatment: a single-institution, 20-year review. JAMA Otolaryngol Head Neck Surg. $2015 ; 141: 313-8$

40. Solomon MC, Vidyasagar MS, Fernandes D, et al. The prognostic implication of the expression of EGFR, p53, cyclin D1, Bcl-2 and p16 in primary locally advanced oral squamous cell carcinoma cases: a tissue microarray study. Med Oncol. 2016;33:138.

41. Ries J, Vairaktaris E, Agaimy A, et al. The relevance of EGFR overexpression for the prediction of the malignant transformation of oral leukoplakia. Oncol Rep. 2013;30:1149-56.

42. Hartmann S, Kipke RU, Rauthe S, et al. Oral brush biopsy and melanoma-associated antigens A (MAGE-A) staining in clinically suspicious lesions. J Craniomaxillofac Surg. 2015;43:2214-8.

43. Hartmann S, Kriegebaum U, Kuchler N, et al. Correlation of MAGE-A tumor antigens and the efficacy of various chemotherapeutic agents in head and neck carcinoma cells. Clin Oral Investig. 2014;18:189-97.

44. Abadie WM, Partington EJ, Fowler CB, et al. Optimal management of proliferative verrucous leukoplakia: a systematic review of the literature. Otolaryngol Head Neck Surg. 2015;153:504-11.

45. Ribeiro AS, Salles PR, da Silva TA, et al. A review of the nonsurgical treatment of oral leukoplakia. Int $\mathrm{J}$ Dent. 2010;2010:186018.

46. Cloitre A, Rosa RW, Arrive E, et al. Outcome of CO2 laser vaporization for oral potentially malignant disorders treatment. Med Oral Pathol Oral Cir Bucal. 2018;23:237-47.

47. Mogedas-Vegara A, Hueto-Madrid JA, Chimenos-Kustner E, et al. Oral leukoplakia treatment with the carbon dioxide laser: a systematic review of the literature. J Cranimaxillofac Surg. 2016;44:331-6.

48. Kumar A, Cascarini L, McCaul JA, et al. How should we manage oral leukoplakia? Br J Oral Maxillofac Surg. 2013;51:377-83.

49. van der Waal I. Potentially malignant disorders of the oral and oropharyngeal mucosa; terminology, classification and present concepts of management. Oral Oncol. 2009;45:317-23.

50. Sang M, Lian Y, Zhou X, et al. MAGE-A family: attractive targets for cancer immunotherapy. Vaccine. 2011;29:8496-8500.

51. Lee AK, Potts PR. A comprehensive guide to the MAGE Family of ubiquitin ligases. J Mol Biol. 2017;429:1114-42. 